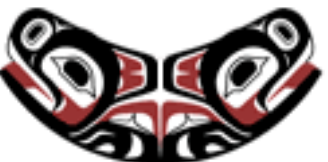

UW Biostatistics Working Paper Series

$5-20-2003$

\title{
Bootstrap Confidence Intervals for Medical Costs With Censored Observations
}

Hongyu Jiang

Harvard University, hjiang@hsph.harvard.edu

Xiao-Hua Zhou

University of Washington, azhou@u.washington.edu

\section{Suggested Citation}

Jiang, Hongyu and Zhou, Xiao-Hua, "Bootstrap Confidence Intervals for Medical Costs With Censored Observations" (May 2003). UW Biostatistics Working Paper Series. Working Paper 202.

http://biostats.bepress.com/uwbiostat/paper202

This working paper is hosted by The Berkeley Electronic Press (bepress) and may not be commercially reproduced without the permission of the copyright holder.

Copyright $(C 2011$ by the authors 


\section{INTRODUCTION}

In a cost-effectiveness study of treatments, the focus is often on the average of total medical costs over a certain time period, say $[0, \tau]$, in a given patient population, where $\tau$ may be five or twenty years. Given the skewed nature of medical cost data, some may argue for the use of the median instead the mean as the measure of central location. However, in analyses of medical cost data, only the mean, not the median, can be used to recover the total medical cost, which reflects the entire expenditure on health care in a given patient population. In this paper, we focus on making inferences on population means.

Estimating this mean can be complicated by censored cost observations for some patients who are not followed for the entire time period $[0, \tau]$. For those censored patients, their true total costs over $[0, \tau]$ are unknown. By considering costs as potentially right-censored survival times, several authors have tried to adjust for censoring by using standard survival techniques. However, Lin et al. [1] have shown this strategy is not valid unless all patients accrue costs with a common rate function over time and have proposed a non-parametric consistent estimator for estimating the mean of the total costs under the assumption the censoring time distribution is discrete. Without making this assumption, Bang and Tsiatis [2] proposed a simple weighted estimator and established its consistency and asymptotic normality. Their estimator works well for lightly censored cost data (censoring rate $\leq 30 \%$ ), but is quite inefficient for heavily censored data (censoring rate $>40 \%$ ) since the estimator does not use any cost information from censored patients. If one can partition $[0, \tau]$ into some subintervals and if piecewise costs of each patient are available over those subintervals up to the observed survival time, Bang and Tsiatis [2] also proposed a more efficient partitioned estimator. Even though asymptotic properties of the partitioned estimator were nicely es- 
tablished, the estimator itself and its asymptotic variance bear some complicated forms. In Section 2, we simplify the expressions of the partitioned estimator and its asymptotic variance so that the simplified versions are computationally more straightforward.

As noted before, medical cost observations have a right skewed distribution (Diehr et al.[3]; Zhou et al. [4]; and Zhou and Tu [5]; Zhou and Tu [6]; and Lumley et al. [7]). In the simulation studies of Bang and Tsiatis [2] and Lin [1], cost observations were generated from some mixed uniform distributions and the resulting total costs have an approximately normal distribution. In this paper we show that confidence intervals based on normal approximations of Bang and Tsiatis' estimators can have much lower than nominal level coverage probabilities when cost observations are moderately or severely skewed. To fix this problem, we propose a bootstrap confidence interval, and our simulation study show that its coverage probabilities are close to nominal level coverage probabilities even for small sample sizes. Our paper is organized as follows. In Section 2 we describe the currently used confidence intervals for the mean of total medical costs when some patients are censored. In Section 3 we propose a bootstrap confidence interval for the mean of total medical costs when some patients are censored. In Section 4 we describe a simulation study for assessing the relative performance of the new bootstrap interval with the existing intervals. In Section 5 we illustrate the proposed method in a real study.

\section{NOTATION AND ESTIMATORS}

Let $M(t)$ denote the random cumulative medical cost of a patient up to time $t$. Since no medical costs will be accrued after death, the total cost of a patient incurred in the time period $[0, \tau]$ is actually $M(T \wedge \tau)$, where $T$ is the true survival time of the patient and $a \wedge b$ 
denotes the minimum of $a$ and $b$. Let us introduce some notation for standard survival data. If we let $C$ denote a random censoring time of the patient, the observed survival time of the patient is $X=T \wedge C$, and $\delta=I(T \leq C)$ indicates whether the true survival time is observed or not, where $I($.$) denotes an indicator function. For a medical cost study, we need to define$ another indicator $\eta=I(T \wedge \tau \leq C)$, which is equal to 1 if the total medical cost accrued over $[0, \tau], M(T \wedge \tau)$, is observed, and 0 if a censored total medical cost, $M(C)$, is observed. In the standard survival analysis, the censoring time, $C$, is assumed to be independent of the true survival time, $T$. For a medical cost study, we need to make a stronger assumption than the one in the standard survival analysis. We have to assume that $C$ is independent of all other random variables in the study; this assumption insures that censoring occurs in a completely random fashion. If the only reason for the occurrence of censoring is the study termination, this assumption holds. This type of censoring is referred to as administrative censoring.

If we do not have the cost history of a patient over time and have only the total cost for each patient, the observed data can be represented as $n$ independent and identically distributed realizations of $(X, \delta, \eta, M(X))$. We can than denote the censored medical cost data as $\left\{\left(X_{i}, \delta_{i}, \eta_{i}, M_{i}\right), i=1 \ldots n\right\}$, where $M_{i}=M\left(X_{i}\right)$. Let $\mu=E(M(T \wedge \tau))$, which is the true mean of the total cost of a patient over time period $[0, \tau]$. Let $K($.$) be the survival$ function of the censoring variable $C$; that is, $K(t)=\operatorname{pr}(C \geq t)$. Bang and Tsiatis [2] proposed the following simple weighted estimator for $\mu$ :

$$
\hat{\mu}_{S}=\frac{1}{n} \sum_{i=1}^{n} \frac{\eta_{i} M_{i}}{\hat{K}\left(T_{i}\right)},
$$

where $\hat{K}($.$) is the Kaplan-Meier (K-M) estimator for K($.$) , based on data \left\{\left(X_{i}, 1-\delta_{i}\right), i=\right.$ $1 \ldots n\}$. Bang and Tsiatis [2] have shown that this estimator is consistent and asymptotically 
normally distributed. They have also provided the asymptotic variance for $n^{1 / 2} \hat{\mu}_{S}$, denoted by $\left(\sigma_{S}\right)^{2}$, and the corresponding consistent estimator $\left(\hat{\sigma}_{S}\right)^{2}$.

Assuming that $\sqrt{n}\left(\hat{\mu}_{S}-\mu\right) / \hat{\sigma}_{S}$ has the standard normal distribution, we may obtain a $(1-\alpha) 100 \%$ confidence interval for $\mu$ as follows:

$$
\left[\hat{\mu}_{S}-n^{-1 / 2} z_{1-\alpha / 2} \hat{\sigma}_{S}, \hat{\mu}_{S}+n^{-1 / 2} z_{1-\alpha / 2} \hat{\sigma}_{S}\right]
$$

where $z_{p}$ denotes the $p$ th quantile of the standard normal distribution. One drawback of this estimator is its efficiency when many patients are censored because we only use cost information from uncensored patients in its calculation.

If we can partition the interval $[0, \tau]$ into $L$ subintervals $\left(t_{j}, t_{j+1}\right], j=0,1, \ldots, L-1$, and if the costs incurred in each of these subintervals up to $X \wedge \tau$ are available for each patient, Bang and Tsiatis [2] also proposed the following more efficient estimator of $\mu$ :

$$
\hat{\mu}_{P}=\frac{1}{n} \sum_{i=1}^{n} \sum_{j=1}^{L} \frac{\eta_{i}^{j} M_{i j}}{\hat{K}_{j}\left(T_{i}^{j}\right)} .
$$

Here $T_{i}^{j}=T_{i} \wedge t_{j}, \eta_{i}^{j}=I\left(T_{i}^{j} \leq C_{i}\right), M_{i j}=M_{i}\left(t_{j}\right)-M_{i}\left(t_{j-1}\right)$, and $\hat{K}_{j}($.$) is the \mathrm{K}-\mathrm{M}$ estimator for the survival function of $C$, based on the dataset $\left\{\left(X_{i}^{j}, 1-\eta_{i}^{j}\right), i=1, \ldots, n\right\}$, where $X_{i}^{j}=T_{i}^{j} \wedge C_{i}$. Let $\lambda^{c}($.$) denote the hazard function of the censoring random variable$ $C_{i}, S_{j}(u)=\operatorname{pr}\left(T_{i}^{j} \geq u\right)$, and

$$
G_{j}\left(M_{l}, u\right)=\frac{1}{S_{j}(u)} E\left\{M_{i l} I\left(T_{i}^{j} \geq u\right)\right\}
$$

Bang and Tsiatis [2] have shown that $n^{\frac{1}{2}}\left(\hat{\mu}_{P}-\mu\right)$ has an asymptotically normal distribution with mean 0 and variance,

$$
\begin{aligned}
\sigma_{P}^{2}= & \operatorname{var}\left(M_{i}-\mu\right)+ \\
& \int_{0}^{\tau} \sum_{j=1}^{L} \sum_{l=1}^{L} S_{j \wedge l}(u)\left\{G_{j \wedge l}\left(M_{j} M_{l}, u\right)-G_{j \wedge l}\left(M_{j}, u\right) G_{j \wedge l}\left(M_{l}, u\right)\right\} \frac{\lambda^{c}(u)}{K(u)} d u .
\end{aligned}
$$


From (2) and (3), we see that to compute $\hat{\mu}_{P}$ and its associated variance we need to compute $L$ times of the estimators $\hat{K}_{j}(),. \hat{S}_{j}($.$) , and \hat{G}_{j}($.$) , which might result in complexity of$ the calculation of variance estimation. In Appendix we show that this partitioned estimator can be simplified to

$$
\hat{\mu}_{P}=\frac{1}{n} \sum_{i=1}^{n} \sum_{j=1}^{L} \frac{\eta_{i}^{j} M_{i j}}{\hat{K}\left(T_{i}^{j}\right)},
$$

where $K($.$) is the Kaplan-Meier estimator for the survival function of C$ based on $\left\{\left(X_{i}, 1-\right.\right.$ $\left.\left.\delta_{i}\right), i=1, \ldots, n\right\}$. In this simplified version, we only need to compute one K-M estimator. Using a different derivation method, we can also show its asymptotic variance can be greatly simplified for computation. The simplified expressions of $\sigma_{P}$ and $\hat{\sigma}_{P}$ are as follows:

$$
\begin{gathered}
\sigma_{P}^{2}=\operatorname{var}\left(M_{i}-\mu\right)+\sum_{j=1}^{L} \sum_{l=1}^{L} \int_{0}^{t_{j \wedge l}} S(u)\left\{G\left(M_{j} M_{l}, u\right)-G\left(M_{j}, u\right) G\left(M_{l}, u\right)\right\} \frac{\lambda^{c}(u)}{K(u)} d u, \\
\hat{\sigma}_{P}^{2}=\frac{1}{n} \sum_{i=1}^{n} \frac{\eta_{i}\left(M_{i}-\hat{\mu}_{P}\right)^{2}}{\hat{K}\left(T_{i}\right)}+\sum_{j=1}^{L} \sum_{l=1}^{L} \int_{0}^{t_{j \wedge l}} \hat{S}(u)\left\{\hat{G}\left(M_{j} M_{l}, u\right)-\hat{G}\left(M_{j}, u\right) \hat{G}\left(M_{l}, u\right)\right\} \frac{d N^{c}(u)}{Y(u) \hat{K}(u)}
\end{gathered}
$$

where

$$
\begin{gathered}
\hat{G}\left(M_{j}, u\right)=\frac{1}{n \hat{S}(u)} \sum_{i=1}^{n} \frac{\eta_{i}^{j} M_{i j} I\left(T_{i} \geq u\right)}{\hat{K}\left(T_{i}^{j}\right)} \\
\hat{G}\left(M_{j} M_{l}, u\right)=\frac{1}{n \hat{S}(u)} \sum_{i=1}^{n} \frac{\eta_{i}^{j \vee l} M_{i j} M_{i l} I\left(T_{i} \geq u\right)}{\hat{K}\left(T_{i}^{j \vee l}\right)} \text {, and } j \vee l=\max (j, l) .
\end{gathered}
$$

Thus, instead of computing $L$ functional estimators of $K_{j}$ 's, $S_{j}$ 's and $G_{j}$ 's as in the original formula for the partitioned estimator and its variance, with the simplified formulas we only need to compute them once, which eases the computation. Assuming the normality for $\sqrt{n}\left(\hat{\mu}_{P}-\mu\right) / \hat{\sigma}_{P}$, we obtain a $(1-\alpha) 100 \%$ confidence interval for $\mu$ as follows:

$$
\left[\hat{\mu}_{P}-n^{-1 / 2} z_{1-\alpha / 2} \hat{\sigma}_{P}, \hat{\mu}_{P}+n^{-1 / 2} z_{1-\alpha / 2} \hat{\sigma}_{P}\right] .
$$

\section{A BOOTSTRAP CONFIDENCE INTERVAL}


Let $\hat{\mu}$ and $(\hat{\sigma})^{2}$ be a simple weighted or partitioned estimator for $\mu$ and its corresponding variance estimator, respectively. Although many bootstrap intervals, including Efron's biascorrected accelerated (BCa) bootstrap interval [8], are available for the population mean, it has been shown that the bootstrap-t interval has the best coverage accuracy in finite sample sizes (Hall [9]; Shao and Tu [10]). Hence we chose to develop a bootstrap-t interval for $\mu$. The bootstrap-t interval is based on the bootstrap distribution of the studentized statistics,

$$
T=\sqrt{n}(\hat{\mu}-\mu) / \hat{\sigma}
$$

By considering the original data, $\left\{\left(X_{i}, \delta_{i}, \eta_{i}, M_{i}\right), i=1, \ldots, n\right\}$, as an independent and identically distributed (i.i.d.) sample from the joint distribution function for $(X, \delta, \eta, M)$, we generate our bootstrap samples, by sampling with replacement from $\left\{\left(X_{i}, \delta_{i}, \eta_{i}, M_{i}\right), i=\right.$ $1, \ldots, n\}$. Let $\left(X_{i}^{* b}, \delta_{i}^{* b}, \eta_{i}^{* b}, M_{i}^{* b}\right), i=1, \ldots, n$, be the $b$ th bootstrap sample, $b=1, \ldots, B$. Let $\hat{\mu}_{b}^{*}$ and $\left(\hat{\sigma}_{b}^{*}\right)^{2}$ be respectively the values of $\hat{\mu}$ and $\hat{\sigma}^{2}$ using the $b$ th bootstrap sample. We calculate the bootstrap version of $T$ as

$$
T_{b}^{*}=\sqrt{n} \frac{\hat{\mu}_{b}^{*}-\hat{\mu}}{\hat{\sigma}_{b}^{*}}
$$

Then, a $(1-\alpha) \%$ bootstrap-t confidence interval for $\mu$ is given by

$$
\left[\hat{\mu}-n^{-1 / 2} U \hat{\sigma}, \hat{\mu}-n^{-1 / 2} L \hat{\sigma}\right]
$$

where $L$ and $U$ are the $\alpha / 2$ th and $(1-\alpha / 2)$ th quantiles of $\left\{T_{b}^{*}, b=1, \ldots, B\right\}$ respectively.

\section{NUMERICAL STUDIES}

We adopted a similar setup for our simulation study as in Bang and Tsiatis [2] except that we used skewed distributions for medical costs. The average 10 -year cost $\mu$ and its $95 \%$ 
confidence interval are parameters of interest. The survival time $T$ was generated from two distributions, the uniform distribution over [0,10 years] and the exponential distribution with a mean of 5 years. We generated an independent censoring variable $C$ from the two uniform distributions, one over [0,20 years] and the other over [0,12.5 years], which correspond to light censoring $(22 \%-25 \%)$ and relatively heavy censoring $(30 \%-40 \%)$ on the survival time within 10 years, respectively. We assumed the true total medical cost of a patient over $[0, T \wedge 10]$, denoted by $M$, had a log-normal distribution; that is, $\log M \sim N\left(\rho, \sigma^{2}\right)$, where $\rho=8+\frac{1}{3} T$ and where $\sigma$ was chosen to be either $0.3,0.5,0.7$ or 1 . Under this setup for the distribution of costs, the expected total medical cost of a patient becomes larger as the survival time increases, and the cost distribution becomes more skewed as $\sigma$ increases. The coefficient of skewness ranged from 1.6 to 8.7 reflecting moderate to severe skewness observed in medical cost data [3-6]. For computing the partitioned estimator, we divided the 10-year period into 10 1-year subintervals and the cost incurred in each subinterval up to $[0, X \wedge 10]$ were obtained according to the cost distribution described above. To construct a bootstrap confidence interval, we resampled 1000 samples with replacement for each generated dataset.

In all the following tables, the top half corresponds to the simulation results when the survival time had a uniform distribution, and the bottom half corresponds to the simulation results when the survival time had an exponential distribution. The first column specifies the parameter $\sigma$ of a log-normal distribution we used for generating medical costs, and the corresponding coefficient of skewness of the cost distribution, $\beta$, is listed in the second column. The third column presents the true expected 10-year total cost $\mu$. In Tables 1-2, since both the simple weighted and partitioned estimates of $\mu$ are more or less unbiased, we did not report the point estimates in the table. For the comparison purpose, we used two 
methods to construct $95 \%$ confidence intervals (C.I.) for $\mu$; the first method uses a normal approximation for Bang and Tsiatis' estimators (denoted as "BT" in the table), and the second method uses the bootstrap method described in Section 3 (denoted as "boot" in the table). $P_{\text {cover }}$ denotes the coverage probability of the C.I.'s.

\section{Table 1 goes here}

All entries in the last eight columns of Table 1 are averages from 1000 simulations with a fixed sample size of 100 in each setting. The simulation results show that for moderate sample sizes, the symmetric confidence intervals based on the normal approximation have coverage probabilities that are much lower than the nominal level when cost distributions are skewed. The problem is more severe as the degree of skewness increases or censoring rate increases. In the presence of light censoring, the improvement in efficiency or coverage accuracy of bootstrap confidence intervals based on the partitioned estimator is minimal compared to those based on the simple weighted estimator. When the censoring is heavy, the former has noticeable efficiency advantage than the latter.

Numerical studies were also conducted at larger sample sizes under the above simulation schemes. Table 2 shows a comparison of the two types of confidence intervals (BT and boot) based on both the simple weighted and the partitioned estimator in the presence of heavy censoring with $n=200$ or 400. As sample size increases, the performance of both types of confidence intervals improve, however, the coverage probabilities from the normal approximation approach are still much lower than the nominal level when the cost distribution is severely skewed. Overall, the bootstrap confidence interval based on the partitioned estimator has the superior performance for sample size $\geq 200$ and censoring rate $\geq 30 \%$. 
Table 2 goes here

For comparison purpose, we also estimated the mean medical cost for the simulated skewed medical cost data with censoring using a simple but very useful estimate in medical cost analysis - mean cost per person-month multiplied by the number of expected follow-up time on study based on the estimated survival distribution. We refer to this estimator as the "person-month estimator" for simplicity. The variance of the person-month estimator is estimated by the empirical variance of the estimates obtained from 1000 bootstrap samples for each simulated dataset and a $95 \%$ confidence interval is constructed using a normal approximation. In Table 3, we present the relative bias $(R B)$ and coverage probability $\left(P_{\text {cover }}\right)$ of the simple estimator under three censoring schemes: no censoring, light censoring and heavy censoring. The relative bias is computed using the difference between the average estimated mean cost and the true mean cost $(\mu)$ divided by the true mean cost. In all settings, the sample size is fixed at 100 and all entries in the last six columns are based on 1000 simulations.

Table 3 goes here

Table 3 shows that the person-month estimate is unbiased when there is no censoring on the survival time. However, the coverage probability may not be correct when the cost distribution is severely skewed $(\beta \geq 4)$. Since the simulated cost processes have increasing monthly cost over time, the person-month estimate tends to underestimate the true mean cost in the presence of censoring and the coverage probabilities of its confidence interval decrease sharply as the censoring rate increases. In other simulations, we found that as long as the cost accumulation rate is uniform over time, the unbiasness of the person-month 
estimator and the coverage accuracy of its confidence intervals are not affected by the failure time distribution or the censoring rate. Thus, for censored medical cost data, if the cost accumulation process is suspected to be non-uniform over time, the simple person-month estimator is not a good choice, and more complicated methods like the simple weighted or partitioned estimator should be used.

\section{A REAL DATA EXAMPLE}

To demonstrate the application of our methodology, we used the same cardiology clinical trial data as in Bang and Tsiatis [2]. In this trial, 10,948 acute coronary syndrome patients were randomized to receive either intravenous bolus and 72-hour eptifibatide (i.e. integrilin) or placebo. The main study objective was to test whether Integrilin could reduce the 30-day incidence of death and myocardial infarction. A prospective economic sub-study with 2547 patients enrolled was also conducted to compare mean medical costs incurred from initiation of the treatment until death or six month after the initiation. Since there is no significant difference in costs across treatments, we use the pooled data to illustrate estimation of the mean cost with censored observations. As there is up to one year delay in reporting the six-month medical cost, we use the cost data available at 550 days after study started to illustrate our methodology and the censoring rate was $48 \%$ at that point. Since this cost-effectiveness study was completed in January 1997, complete cost data for all enrolled patients are available now. The sample average of the mean cost is $\$ 32,596$, which can be considered as the true mean cost and be compared with the estimates we obtained from the censored medical cost data. As piece-wise costs over time were not available, the partitioned estimator was not used in estimating the mean medical cost. Simple weighted estimator 
gives an estimate of the mean six-month medical cost as $\$ 29,301$. The bootstrap confidence interval for the mean six-month cost based on 5000 resampling is $[\$ 25,424, \$ 34,016]$ while the confidence interval constructed using normal approximation is $[\$ 26,406, \$ 32,195]$. Not surprisingly, the bootstrap confidence interval is wider than the interval based on the normal approximation, and the former covers the true mean cost $(\$ 32,596)$ while the latter does not. The result is consistent with our simulation results which showed that the normal approximation interval has coverage probability that is much lower than the nominal level while the bootstrap interval has the coverage probability that is close to the nominal level. On the other hand, the person-month estimator gives an estimate of $\$ 40,855$ for the mean six-month medical cost and a confidence interval $[\$ 39,236, \$ 42,474]$. The person-month estimator overestimates the true mean because in this dataset, patients with shorter survival times tend to have higher medical costs.

\section{CONCLUSIONS}

Cost-effectiveness studies are often conducted in comparing treatments for life threatening diseases. Mean cost incurred from the start of a treatment till death or certain timepoint after the implementation of treatment is frequently of interest. In most cases, cost data may be censored to certain degree due to limited followup time. On the other hand, the cost accumulation rate over time may depend on disease progression and the survival time, and hence is non-uniform over time. Thus, the simple person-month estimator is no longer appropriate and special estimates as proposed by Bang \& Tsiatis should be used. In this article, we simplified the expression of Bang \& Tsiatis' partitioned estimator to make the computation procedure much easier, and proposed a bootstrap confidence interval for the 
mean of medical costs with censored observations instead of the currently available normal approximation confidence intervals. Numerical studies have shown that the proposed bootstrap confidence intervals have much better coverage accuracy than the normal approximation ones when medical costs have a skewed distribution. When there is light censoring on medical costs $(\leq 25 \%)$, we have found that the bootstrap confidence intervals based on the simple weighted estimator is preferred due to its simplicity and good coverage accuracy. For heavily censored cost data (censoring rate $\geq 30 \%$ ) with larger sample sizes $(n \geq 200)$, the bootstrap confidence intervals based on the partitioned estimator has superior performance in terms of both efficiency and coverage accuracy.

Our proposed bootstrap sampling was based on one simple sampling plan for a Cox's regression model that has been shown to have some good theoretical properties (Shao and Tu [9]). However, there are other types of bootstrap procedures available for survival data (Burr [10]), such as the one based on generalized residuals (Shao and Tu [9]) and the one based on conditional resampling (Davison and Hinkley [11]). It is an interesting future research topic to study both theoretical and finite-sample properties of these various bootstrap methods for the analysis of health care costs.

\section{ACKNOWLEDGEMENT}

This work was supported in part by Agency for Healthcare Quality and Research (AHRQ) and NIH grants R01HS013105 and R01AI24643. We would like to thank Dr. Bang for providing us the dataset used in the paper. We also like to thank two reviewers for their helpful comments that have resulted an improved version of the paper.

\section{APPENDIX}


According to Bang and Tsiatis [2], the partitioned estimator $\hat{\mu}_{P}$ is as given in (2) and the asymptotic variance of $n^{\frac{1}{2}}\left(\hat{\mu}_{P}-\mu\right)$ has following form:

$$
\begin{aligned}
\sigma_{P}^{2}= & \operatorname{var}\left(M_{i}-\mu\right)+ \\
& \int_{0}^{\tau} \sum_{j=1}^{L} \sum_{l=1}^{L} S_{j \wedge l}(u)\left\{G_{j \wedge l}\left(M_{j} M_{l}, u\right)-G_{j \wedge l}\left(M_{j}, u\right) G_{j \wedge l}\left(M_{l}, u\right)\right\} \frac{\lambda^{c}(u)}{K(u)} d u .
\end{aligned}
$$

An estimator of $\sigma_{P}$ is proposed as

$$
\begin{aligned}
\hat{\sigma}_{P}^{2}= & \frac{1}{n} \sum_{i=1}^{n} \frac{\eta_{i}\left(M_{i}-\hat{\mu}_{P}\right)^{2}}{\hat{K}\left(T_{i}\right)}+ \\
& \int_{0}^{\tau} \sum_{j=1}^{L} \sum_{l=1}^{L} \hat{S}_{j \wedge l}(u)\left\{\hat{G}_{j \wedge l}\left(M_{j} M_{l}, u\right)-\hat{G}_{j \wedge l}\left(M_{j}, u\right) \hat{G}_{j \wedge l}\left(M_{l}, u\right)\right\} \frac{d N^{c}(u)}{Y(u) \hat{K}(u)},
\end{aligned}
$$

where

$$
\begin{aligned}
\hat{G}_{j \wedge l}\left(M_{l}, u\right) & =\frac{1}{n \hat{S}_{j \wedge l}(u)} \sum_{i=1}^{n} \frac{\eta_{i}^{j \vee l} M_{i l} I\left(T_{i}^{j \wedge l} \geq u\right)}{\hat{K}_{j \vee l}\left(T_{i}^{j \vee l}\right)}, \\
\hat{G}_{j \wedge l}\left(M_{j} M_{l}, u\right) & =\frac{1}{n \hat{S}_{j \wedge l}(u)} \sum_{i=1}^{n} \frac{\eta_{i}^{j \vee l} M_{i j} M_{i l} I\left(T_{I}^{j \wedge l} \geq u\right)}{\hat{K}_{j \vee l}\left(T_{i}^{j \vee l}\right)},
\end{aligned}
$$

$N^{c}(u)=\sum_{i=1}^{n} I\left(X_{i} \leq u, \delta_{i}=0\right), Y(u)=\sum_{i=1}^{n} I\left(X_{i} \geq u\right)$, and $\hat{S}_{j}($.$) is the K-M estimator of$ $S_{j}(\cdot)$

The simplified partitioned estimator is :

$$
\hat{\mu}_{P}=\frac{1}{n} \sum_{i=1}^{n} \sum_{j=1}^{L} \frac{\eta_{i}^{j} M_{i j}}{\hat{K}\left(T_{i}^{j}\right)} .
$$

Since it can be shown that

$$
\hat{K}_{j}(t)=\left\{\begin{array}{ll}
\hat{K}(t) & \text { if } \quad t \leq t_{j} \\
\hat{K}\left(t_{j}\right) & \text { if } \quad t>t_{j}
\end{array},\right.
$$

and we only need to evaluate $\hat{K}_{j}$ at $T_{i}^{j}$ which does not exceed $t_{j}$, we can drop the subscript $j$ from $\hat{K}_{j}$ and use only one version instead of $L$ versions of functional estimates of $K($.$) in$ the computation. 
Furthermore, we can simplify the expressions in (3) and (5) by noticing that $I\left(t_{j} \geq u\right)$ is just a constant for any time point $u$. Hence,

$$
S_{j}(u)=\operatorname{pr}\left\{T_{i}^{j}>u\right\}=\operatorname{pr}\left\{T_{i}>u\right\} I\left(t_{j} \geq u\right)=S(u) I\left(t_{j} \geq u\right)
$$

where $S(u)=\operatorname{pr}(T>u)$, and

$$
E\left\{M_{i j} I\left(T_{i}^{j} \geq u\right)\right\}=E\left\{M_{i j} I\left(T_{i} \geq u\right)\right\} I\left(t_{j} \geq u\right) .
$$

This implies that

$$
\begin{aligned}
G_{j}\left(M_{l}, u\right) & =\frac{1}{S_{j}(u)} E\left\{M_{i l} I\left(T_{i}^{j} \geq u\right)\right\} \\
& =\frac{I\left(t_{j} \geq u\right)}{S(u)} E\left\{M_{i l} I\left(T_{i} \geq u\right)\right\} \equiv G\left(M_{l}, u\right) I\left(t_{j} \geq u\right) .
\end{aligned}
$$

The simplified expressions of $\sigma_{P}$ and $\hat{\sigma}_{P}$ are as follows:

$$
\begin{gathered}
\sigma_{P}=\operatorname{var}\left(M_{i}-\mu\right)+\sum_{j=1}^{L} \sum_{l=1}^{L} \int_{0}^{t_{j \wedge l}} S(u)\left\{G\left(M_{j} M_{l}, u\right)-G\left(M_{j}, u\right) G\left(M_{l}, u\right)\right\} \frac{\lambda^{c}(u)}{K(u)} d u, \\
\hat{\sigma}_{P}=\frac{1}{n} \sum_{i=1}^{n} \frac{\eta_{i}\left(M_{i}-\hat{\mu}_{P}\right)^{2}}{\hat{K}\left(T_{i}\right)}+\sum_{j=1}^{K} \sum_{l=1}^{K} \int_{0}^{t_{j \wedge l}} \hat{S}(u)\left\{\hat{G}\left(M_{j} M_{l}, u\right)-\hat{G}\left(M_{j}, u\right) \hat{G}\left(M_{l}, u\right)\right\} \frac{d N^{c}(u)}{Y(u) \hat{K}(u)}
\end{gathered}
$$

where

$$
\begin{gathered}
\hat{G}\left(M_{j}, u\right)=\frac{1}{n \hat{S}(u)} \sum_{i=1}^{n} \frac{\eta_{i}^{j} M_{i j} I\left(T_{i} \geq u\right)}{\hat{K}\left(T_{i}^{j}\right)} \quad \text { and } \\
\hat{G}\left(M_{j} M_{l}, u\right)=\frac{1}{n \hat{S}(u)} \sum_{i=1}^{n} \frac{\eta_{i}^{j \vee l} M_{i j} M_{i l} I\left(T_{i} \geq u\right)}{\hat{K}\left(T_{i}^{j \vee l}\right)}
\end{gathered}
$$

\section{References}

[1] Lin DY, Feuer EJ, Etzioni R, Wax, Y. Estimating medical costs from incomplete followup data. Biometrics 1997; 53:419-434. 
[2] Bang H, Tsiatis AA. Estimating medical costs with censored data. Biometrika 2000; 87:329-343.

[3] Diehr P, Yanez D, Ash A, Hornbrook M, Lin DY. Methods for analyzing health care utilization and costs. Annual Reviews of Public Health 1999; 20:125-144.

[4] Zhou XH, Melfi CA, Hui SL. Methods for comparison of costs data Annals of Internal Medicine 1997; 127:752-756.

[5] Zhou XH, Tu W. Comparison of several population means when their samples contain non-zero log-normal and possibly zero observations. Biometrics 1999; 55:645-651.

[6] Zhou XH, Tu W. Confidence intervals for the mean of diagnostic test charge data containing zeros. Biometrics 2000; 56:1118-1125.

[7] Lumley T, Diehr P, Emerson S, and Chen L. The importance of the normality assumption in large public health data sets. Annual Review of Public Health 2002; 23:151-169.

[8] Efron B, Tibshirani R. An introduction to the bootstrap. New York: Chapman \& Hall Ltd, 1993.

[9] Hall P. The Bootstrap and Edgeworth Expansion. New York: Springer-Verlag, 1992.

[10] Shao J, Tu D. The Jackknife and Bootstrap. New York: Springer-Verlag, 1995.

[11] Burr D. A comparison of certain bootstrap confidence intervals in the Cox model. Journal of the American Statistical Association 1994; 89:1290-1320.

[12] Davison AC, and Hinkley DV. Bootstrap Methods and Their Application. Cambridge, U.K.: Cambridge University Press, 1997. 
Table 1: Simulation results for bootstrap and BT confidence intervals $(n=100)$

\begin{tabular}{|c|c|c|c|c|c|c|c|c|c|c|}
\hline \multirow[b]{3}{*}{$\sigma$} & \multirow[b]{3}{*}{$\beta$} & \multirow[b]{3}{*}{$\mu$} & \multicolumn{4}{|c|}{ Light Censoring } & \multicolumn{4}{|c|}{ Heavy Censoring } \\
\hline & & & \multicolumn{2}{|c|}{ C.I. width } & \multicolumn{2}{|c|}{$100 * P_{\text {cover }}$} & \multicolumn{2}{|c|}{ C.I. width } & \multicolumn{2}{|c|}{$100 * P_{\text {cover }}$} \\
\hline & & & $\mathrm{BT}$ & boot & $\mathrm{BT}$ & boot & $\mathrm{BT}$ & boot & $\mathrm{BT}$ & boot \\
\hline \multicolumn{11}{|c|}{ Uniform survival distribution } \\
\hline 0.3 & 1.64 & 25287 & & & & & & & & \\
\hline \multicolumn{3}{|c|}{ Simple weighted estimate } & 11240 & 12719 & 93.5 & 95.7 & 12899 & 18544 & 89.0 & 93.4 \\
\hline \multicolumn{3}{|c|}{ Partitioned estimate } & 10949 & 12150 & 94.0 & 95.8 & 12001 & 15213 & 91.0 & 93.8 \\
\hline \multicolumn{3}{|c|}{$\begin{array}{lll}0.5 & 2.60 & 27393\end{array}$} & & & & & & & & \\
\hline \multicolumn{3}{|c|}{ Simple weighted estimate } & 14223 & 16992 & 90.9 & 93.2 & 16980 & 27179 & 85.8 & 93.2 \\
\hline \multicolumn{3}{|c|}{ Partitioned estimate } & 13713 & 16192 & 92.3 & 94.7 & 14975 & 20546 & 89.4 & 93.0 \\
\hline \multicolumn{3}{|c|}{$\begin{array}{lll}0.7 & 4.20 & 30885\end{array}$} & & & & & & & & \\
\hline \multicolumn{3}{|c|}{ Simple weighted estimate } & 19310 & 25634 & 89.6 & 92.2 & 23283 & 44121 & 84.0 & 92.4 \\
\hline \multicolumn{3}{|c|}{ Partitioned estimate } & 18274 & 23511 & 90.6 & 92.3 & 19628 & 30075 & 86.5 & 92.9 \\
\hline \multicolumn{11}{|c|}{$\begin{array}{lll}1 & 7.87 & 39856\end{array}$} \\
\hline \multicolumn{3}{|c|}{ Simple weighted estimate } & 34660 & 559331 & 85.6 & 91.7 & 40176 & 96377 & 80.4 & 89.9 \\
\hline \multicolumn{3}{|c|}{ Partitioned estimate } & 31628 & 52694 & 88.0 & 93.5 & 32089 & 58407 & 82.3 & 91.3 \\
\hline \multicolumn{11}{|c|}{ Exponential survival distribution } \\
\hline \multicolumn{11}{|c|}{$\begin{array}{lll}0.3 & 1.87 & 24896\end{array}$} \\
\hline \multicolumn{3}{|c|}{ Simple weighted estimate } & 14133 & 16365 & 93.8 & 95.8 & 16274 & 38301 & 86.1 & 95.3 \\
\hline \multicolumn{3}{|c|}{ Partitioned estimate } & 13859 & 15831 & 91.6 & 94.7 & 15220 & 22919 & 90.5 & 92.8 \\
\hline \multicolumn{3}{|c|}{$\begin{array}{lll}0.5 & 2.84 & 26970\end{array}$} & & & & & & & & \\
\hline \multicolumn{3}{|c|}{ Simple weighted estimate } & 17673 & 22379 & 90.2 & 94.5 & 20834 & 54970 & 83.6 & 93.5 \\
\hline \multicolumn{3}{|c|}{ Partitioned estimate } & 16980 & 20864 & 91.4 & 93.8 & 18698 & 30610 & 86.9 & 89.7 \\
\hline \multicolumn{3}{|c|}{$\begin{array}{lll}0.7 & 4.55 & 30408\end{array}$} & & & & & & & & \\
\hline Sim & reighte & timate & 23576 & 34009 & 88.4 & 94.6 & 28005 & 92673 & 80.3 & 92.3 \\
\hline Par & led est & & 21779 & 30030 & 89.0 & 94.2 & 23500 & 47899 & 83.1 & 93.2 \\
\hline 1 & 8.72 & 39240 & & & & & & & & \\
\hline Sim & reighte & timate & 39148 & 73360 & 84.0 & 92.3 & 47460 & 215131 & 75.3 & 88.9 \\
\hline Par & led est & & 36477 & 65231 & 84.2 & 90.9 & 37398 & 86788 & 77.9 & 88.3 \\
\hline
\end{tabular}


Table 2: Simulation results for bootstrap and BT confidence intervals under heavy censoring

\begin{tabular}{|c|c|c|c|c|c|c|c|c|c|c|}
\hline \multirow[b]{3}{*}{$\sigma$} & \multirow[b]{3}{*}{$\beta$} & \multirow[b]{3}{*}{$\mu$} & \multicolumn{4}{|c|}{$n=200$} & \multicolumn{4}{|c|}{$n=400$} \\
\hline & & & \multicolumn{2}{|c|}{ C.I. width } & \multicolumn{2}{|c|}{$100 * P_{\text {cover }}$} & \multicolumn{2}{|c|}{ C.I. width } & \multicolumn{2}{|c|}{$100 * P_{\text {cover }}$} \\
\hline & & & $\mathrm{BT}$ & boot & $\mathrm{BT}$ & boot & $\mathrm{BT}$ & boot & $\mathrm{BT}$ & boot \\
\hline \multicolumn{11}{|c|}{ Uniform survival distribution } \\
\hline 0.3 & 1.64 & 25287 & & & & & & & & \\
\hline \multicolumn{3}{|c|}{ Simple weighted estimate } & 9449 & 11361 & 91.3 & 94.0 & 6799 & 7384 & 93.0 & 93.8 \\
\hline \multicolumn{3}{|c|}{ Partitioned estimate } & 8784 & 9861 & 92.7 & 95.2 & 6305 & 6650 & 93.5 & 94.5 \\
\hline & 2.60 & 27393 & & & & & & & & \\
\hline \multicolumn{3}{|c|}{ Simple weighted estimate } & 12697 & 16424 & 89.4 & 92.3 & 9222 & 10576 & 93.6 & 93.8 \\
\hline \multicolumn{3}{|c|}{ Partitioned estimate } & 11074 & 13128 & 91.6 & 93.2 & 7993 & 8771 & 94.1 & 94.3 \\
\hline \multicolumn{11}{|c|}{$\begin{array}{lll}0.7 & 4.20 & 30885\end{array}$} \\
\hline \multicolumn{3}{|c|}{ Simple weighted estimate } & 17534 & 24580 & 88.7 & 93.2 & 13243 & 16460 & 91.2 & 94.8 \\
\hline \multicolumn{3}{|c|}{ Partitioned estimate } & 14689 & 18698 & 90.0 & 93.4 & 10901 & 12660 & 92.4 & 94.6 \\
\hline \multicolumn{11}{|c|}{$\begin{array}{lll}1 & 7.87 & 39856\end{array}$} \\
\hline \multicolumn{3}{|c|}{ Simple weighted estimate } & 31007 & 53530 & 85.0 & 91.4 & 24241 & 35787 & 88.0 & 90.9 \\
\hline \multicolumn{3}{|c|}{ Partitioned estimate } & 25347 & 38389 & 86.7 & 93.3 & 19273 & 25619 & 89.2 & 92.7 \\
\hline \multicolumn{11}{|c|}{ Exponential survival distribution } \\
\hline \multicolumn{11}{|c|}{$\begin{array}{lll}0.3 & 1.87 & 24896\end{array}$} \\
\hline \multicolumn{3}{|c|}{ Simple weighted estimate } & 12079 & 16667 & 93.0 & 96.3 & 8753 & 9408 & 93.6 & 93.8 \\
\hline \multicolumn{3}{|c|}{ Partitioned estimate } & 11133 & 13160 & 93.3 & 95.9 & 8017 & 8452 & 94.4 & 94.5 \\
\hline \multicolumn{11}{|c|}{$\begin{array}{lll}0.5 & 2.84 & 26970\end{array}$} \\
\hline \multicolumn{3}{|c|}{ Simple weighted estimate } & 16091 & 25994 & 88.0 & 93.6 & 11798 & 13829 & 93.2 & 94.6 \\
\hline \multicolumn{3}{|c|}{ Partitioned estimate } & 13700 & 17564 & 90.2 & 93.0 & 9978 & 11090 & 93.0 & 93.7 \\
\hline \multicolumn{11}{|c|}{$\begin{array}{lll}0.7 & 4.55 & 30408\end{array}$} \\
\hline \multicolumn{3}{|c|}{ Simple weighted estimate } & 22610 & 42025 & 86.7 & 93.3 & 16981 & 22730 & 91.0 & 94.2 \\
\hline Par & ied est & & 18329 & 26470 & 89.3 & 93.5 & 13559 & 16576 & 91.8 & 94.4 \\
\hline 1 & 8.72 & 39240 & & & & & & & & \\
\hline Sim & reighte & timate & 37738 & 88373 & 82.2 & 91.2 & 30418 & 50353 & 86.7 & 91.5 \\
\hline Par & ed est & & 29737 & 52229 & 85.8 & 92.3 & 23251 & 33599 & 88.1 & 92.7 \\
\hline
\end{tabular}


Table 3: Simulation results for person-month estimate

\begin{tabular}{|c|c|c|c|c|c|c|c|c|}
\hline \multirow[b]{2}{*}{$\sigma$} & \multirow[b]{2}{*}{$\beta$} & \multirow[b]{2}{*}{$\mu$} & \multicolumn{2}{|c|}{ no censoring } & \multicolumn{2}{|c|}{ light censoring } & \multicolumn{2}{|c|}{ heavy censoring } \\
\hline & & & $100 * R B$ & $100 * P_{\text {cover }}$ & $100 * R B$ & $100 * P_{\text {cover }}$ & $100 * R B$ & $100 * P_{\text {cover }}$ \\
\hline \multicolumn{9}{|c|}{ Uniform survival distribution } \\
\hline 0.3 & 1.64 & 25287 & 0.2 & 93.1 & -8.4 & 80.7 & -15.6 & 55.6 \\
\hline 0.5 & 2.60 & 27393 & 0.3 & 93.9 & -8.7 & 79.5 & -15.1 & 60.9 \\
\hline 0.7 & 4.20 & 30885 & 0.2 & 92.0 & -9.3 & 78.5 & -15.5 & 64.0 \\
\hline 1 & 7.87 & 39856 & 0.1 & 89.3 & -8.0 & 77.4 & -14.6 & 66.6 \\
\hline \multicolumn{9}{|c|}{ Exponential survival distribution } \\
\hline 0.3 & 1.87 & 24896 & 0.3 & 94.2 & -10.8 & 78.6 & -20.4 & 51.1 \\
\hline 0.5 & 2.84 & 26970 & 0.4 & 93.3 & -11.5 & 77.6 & -20.4 & 53.7 \\
\hline 0.7 & 4.55 & 30408 & -1.3 & 89.5 & -11.7 & 75.4 & -21.5 & 54.8 \\
\hline 1 & 8.72 & 39240 & 0.5 & 88.4 & -10.5 & 73.9 & -19.9 & 60.2 \\
\hline
\end{tabular}

